\title{
QCD analysis of light charged Higgs production through polarized top quark decay in two different frames
}

\section{S. Mohammad Moosavi Nejad ${ }^{a, b}$ and S. Abbaspour ${ }^{a}$}

\author{
${ }^{a}$ Faculty of Physics, Yazd University, \\ P.O. Box 89195-741, Yazd, Iran \\ ${ }^{b}$ School of Particles and Accelerators, Institute for Research in Fundamental Sciences (IPM), \\ P.O. Box 19395-5531, Tehran, Iran
}

E-mail: mmoosavi@yazd.ac.ir, abbaspoor_516@yahoo.com

\begin{abstract}
Light and heavy charged Higgs bosons are predicted by many models with an extended Higgs sector such as the two-Higgs-doublet model (2HDM). Searches for the charged Higgs bosons have been done by the ATLAS and the CMS experiments at the Large Hadron Collider (LHC) in proton-proton collision. However, a definitive search of charged Higgs bosons still has to be carried out by the LHC experiments. The experimental observation of charged Higgs bosons would indicate physics beyond the Standard Model. In the present work we study the $\mathcal{O}\left(\alpha_{s}\right)$ correction to the energy spectrum of the inclusive bottom-flavored mesons $\left(X_{b}\right)$ in polarized top quark decays into a light charged Higgs boson $\left(m_{H^{+}}<m_{t}\right)$ and a massless bottom quark followed by the hadronization process $b \rightarrow X_{b}$ in the type-I 2HDM, i.e. $t(\uparrow) \rightarrow H^{+} b \rightarrow H^{+} X_{b}+$ Jet. This spin-dependent energy distribution is studied in two different helicity coordinate systems. This study could be considered as a new channel to indirect search for the charged Higgs bosons. To present our phenomenological predictions, we restrict ourselves to the constraints on the $m_{H^{+}}-\tan \beta$ parameter space determined by the recent results of the CMS and the ATLAS collaborations.
\end{abstract}

KEYWORDS: Supersymmetry Phenomenology

ARXIV EPRINT: 1611.08017 


\section{Contents}

1 Introduction 1

2 Formalism 3

3 Analytic results for $d \hat{\Gamma} / d x_{i} \quad 5$

3.1 Born-level rate of $t \rightarrow b H^{+}$in ZM-VFNS 6

$\begin{array}{lll}3.2 & \text { Virtual corrections } & 7\end{array}$

3.3 Real gluon corrections 8

3.4 Analytic results for partial decay rates $d \hat{\Gamma} / d x_{i}$ in the helicity system $2 \quad 9$

4 Numerical analysis in type-I 2HDM 11

5 Conclusions 16

\section{Introduction}

Charged Higgs bosons are predicted by several non-minimal Higgs scenarios [1], such as models including Higgs triplets [2] and two-Higgs-doublet models (2HDM) [3]. In the 2HDM, as a simplest model, the Higgs sector of the Standard Model (SM) is extended typically by adding an extra doublet of complex Higgs fields. In this model, after spontaneous symmetry breaking the particle spectrum includes five physical Higgs bosons: light and heavy CP-even Higgs bosons $\mathrm{h}$ and $\mathrm{H}$ with $m_{H}>m_{h}$, a CP-odd Higgs boson A, plus two charged Higgs bosons $H^{ \pm}$[4]. The discovery of a charged Higgs boson would clearly indicate unambiguous evidence for the presence of new physics beyond the SM.

The production and decay modes of charged Higgs bosons depend on their masses, $m_{H^{ \pm}}$. At hadron colliders, charged Higgs bosons can be produced in several channels. In a type-I or type-II 2HDM, which type-II is the Higgs sector of the Minimal Supersymmetric Standard Model (MSSM) up to SUSY corrections [5], the main production mode at the Large Hadron Collider (LHC) for light charged Higgs (with $m_{H^{ \pm}}<m_{t}$ ) is through the top quark decay $t \rightarrow b H^{+}$. In this case, the light charged Higgses are produced most frequently via $t \bar{t}$ production. At the LHC, a cross section of $\sigma(p p \rightarrow t \bar{t} X) \approx 1(\mathrm{nb})$ is expected at design energy $\sqrt{S}=14 \mathrm{TeV}[6]$. With the LHC design luminosity of $10^{34} \mathrm{~cm}^{-2} \mathrm{~s}^{-1}$ in each of the four experiments, it is expected to produce about 90 million $t \bar{t}$-pairs per year $[7,8]$. Thus, the LHC is a superlative top factory. At the LHC, the charged Higgs bosons are searched for in the subsequent decay products of the top pairs $t \bar{t} \rightarrow H^{ \pm} W^{\mp} b \bar{b}$ and $t \bar{t} \rightarrow H^{ \pm} H^{\mp} b \bar{b}$ when $H^{ \pm}$decays into $\tau$-lepton and neutrino. For a review of all available production modes of light charged Higgs at the LHC, see also [9].

The combined Large Electron-Positron (LEP) experiments have determined a lower limit for the charged Higgs mass in a type-II 2HDM with $B\left(H^{+} \rightarrow \tau \nu\right)=1$ as $m_{H^{+}}>$ 
$94 \mathrm{GeV}$ [10], and the lower limit for any $B\left(H^{+} \rightarrow \tau \nu\right)$ as $80 \mathrm{GeV}$. The experimental results from the Tevatron placed upper limits on $B\left(t \rightarrow H^{+} b\right)$ in the 15-20\% range for light charged Higgs bosons. Both the CMS [11] and ATLAS $[12,13]$ collaborations searched for light charged Higgs bosons assuming $B\left(H^{+} \rightarrow \tau \nu\right)=1$ and improved the Tevatron limits to the $1-4 \%$ range for a mass range $m_{H^{+}}=90-160 \mathrm{GeV}$. We will discuss about the recent results on a search for the charged Higgs bosons by the CMS [14, 15] and ATLAS [16, 17] collaborations when we present our numerical analysis in section 4.

The primary purpose of the present manuscript is the evaluation of the order $\alpha_{s}$ QCD corrections to the differential decay width $\left(d \hat{\Gamma} / d x_{i}\right)$ of a polarized top quark into a charged Higgs boson and a bottom quark, $t(\uparrow) \rightarrow b H^{+}$, where $x_{i}$ is the scaled-energy fraction of the b-quark or the gluon emitted at the next-to-leading order (NLO). In the next section, we shall explain that to obtain the energy distribution of hadrons produced through the top decays in the $2 \mathrm{HDM}$, one needs these differential decay widths. The NLO QCD corrected decay distributions, $\Gamma\left(t \rightarrow b H^{+}\right)$, were previously computed in [18] for the polarized top quarks, and in [19-22] for the unpolarized ones. In refs. [23, 24], we calculated the unpolarized differential decay width $d \hat{\Gamma}\left(t \rightarrow b H^{+}\right) / d x_{b}$ at NLO and showed that our result after integration over $x_{b}\left(0 \leq x_{b} \leq 1\right)$ is in complete agreement with refs. [19-21] and the corrected version of [22]. In [25], we studied the $\mathcal{O}\left(\alpha_{s}\right)$ radiative corrections to the spindependent differential decay rate of the process $t(\uparrow) \rightarrow b H^{+}$in a special helicity coordinate system with the event plane defined in the $(x, z)$ plane and the z-axis along the Higgs boson three-momentum (in the following called system 1). In this frame, the top quark polarization vector was measured with respect to the direction of the Higgs 3-momentum. We checked that our result was in complete agreement with the result presented in [18] after integration over $x_{b}\left(0 \leq x_{b} \leq 1\right)$.

Generally, to define the planes one needs to measure the momentum directions of the momenta $\vec{p}_{b}$ and $\vec{p}_{H^{+}}$and the polarization direction of the top quark, where the measurement of the momentum direction of $\vec{p}_{b}$ requires the use of a jet finding algorithm, whereas the polarization direction of the top quark must be obtained from the theoretical input. For example, in $e^{+} e^{-}$interactions the polarization degree of the top quark can be tuned with the help of polarized beams.

In the present work, we analyze the angular distribution of differential width of the process $t(\uparrow) \rightarrow b H^{+}$in a different helicity coordinate system where, as before, the event plane is the $(\mathrm{x}, \mathrm{z})$ plane but with the z-axis along the bottom quark (in the following called system 2). In this system, the polarization direction of the top quark is evaluated with respect to the b-quark three-momentum (z-axis). This result is completely new. We also calculate the decay width $\Gamma\left(t(\uparrow) \rightarrow b H^{+}\right)$in this new frame by integrating $d \Gamma / d x_{b}$ over $0 \leq x_{b} \leq 1$ and compare it with the previous result from [18].

On the other hand, bottom quarks produced through the top decays hadronize $(b \rightarrow$ $X_{b}$ ) before they decay, therefore, each b-jet $X_{b}$ contains a bottom-flavored hadron which most of the times is a B-meson. At the LHC, of particular interest is the distribution in the scaled-energy of B-mesons $\left(x_{B}\right)$ produced through $t(\uparrow) \rightarrow B H^{+}+X$ in the top quark rest frame. The study of these energy distributions in the polarized and unpolarized top decays could be proposed as a new channel to indirect search for the charged Higgs bosons 
at the LHC. In [23, 24], we studied the energy spectrum of the bottom-flavored mesons in unpolarized top quark decays into a charged Higgs boson and a bottom quark at NLO in the 2HDM. In [25] we studied the spin-dependent energy distribution of B-mesons produced through the polarized top decays at NLO in the helicity coordinate system 1. Here, our specific purpose is to study this angular correlation in a different helicity frame (system 2). Through this paper, we present our predictions for the B-meson energy spectrum in the polarized and unpolarized top decays and shall compare the polarized results in both helicity systems 1 and 2 .

In the SM, due to the element $\left|V_{t b}\right| \approx 1$ of the Cabibbo-Kobayashi-Maskawa (CKM) [26, 27] quark mixing matrix, the top quark decays dominantly through the two-body mode $t \rightarrow b W^{+}$. In [28-32], we investigated the energy distribution of B-mesons produced in polarized and unpolarized top quark decays in the SM. By ignoring the interference contribution between the SM top decay, and the 2HDM top decay into charged Higgs, in each top decay (polarized or unpolarized) to obtain the total distribution of the B-hadron energy two contributions due to the decay modes $t \rightarrow b H^{+}$(in the 2HDM) and $t \rightarrow b W^{+}$ (in the SM) should be summed up. Indeed, for the unpolarized top quark decay one has; $d \Gamma_{\text {tot }} / d x_{B}=d \Gamma^{\mathrm{SM}}\left(t \rightarrow B W^{+}\right) / d x_{B}+d \Gamma^{\mathrm{BSM}}\left(t \rightarrow B H^{+}\right) / d x_{B}$. The same result is valid for the polarized top decay as long as the spin direction of the polarized top quark is evaluated relative to the b-quark three-momentum. Thus, at the LHC any deviation of the B-meson energy spectrum from the SM predictions can be considered as a signal for the existence of charged Higgs. Although, the SM contribution is normally larger than the one coming from $2 \mathrm{HDM}[23,24]$, but there is always a clear separation between the decay channels $t \rightarrow b W^{+}$ and $t \rightarrow b H^{+}$in both the $t \bar{t} X$ pair production and the $t / \bar{t} X$ single top production at the LHC, this point is mentioned in [33].

This paper is organized as follows. In section 2, we study the inclusive production of a meson from polarized top quark considering the factorization theorem and DGLAP equations. In section 3, we present our analytical results of the $\mathcal{O}\left(\alpha_{S}\right)$ QCD corrections to the tree-level rate of $t(\uparrow) \rightarrow b H^{+}$. In section 4 , we present our numerical analysis of inclusive production of a meson from polarized top quark decay considering two different helicity coordinate systems. In section 5 , our conclusions are summarized.

\section{Formalism}

In the proposed way to search for the light charged Higgs bosons, we study the inclusive production of a bottom-flavored meson (B) from polarized top quark decay in the following process

$$
t(\uparrow) \rightarrow b H^{+}(g) \rightarrow H^{+} B+X,
$$

where $\mathrm{X}$ stands for the unobserved final states and the gluon contributes to the real radiation at NLO. Both the b-quark and the gluon may hadronize into the B-meson.

If we label the four-momenta of top quark,b-quark, gluon and B-meson by $p_{t}, p_{b}, p_{g}$ and $p_{B}$, respectively, then in the top quark rest frame the b-quark, gluon, and B-meson take energies $E_{i}=p_{t} \cdot p_{i} / m_{t}(\mathrm{i}=\mathrm{b}, \mathrm{g}, \mathrm{B})$, where $m_{B} \leq E_{B} \leq\left(m_{t}^{2}+m_{B}^{2}-m_{H^{+}}^{2}\right) /\left(2 m_{t}\right), m_{b} \leq$ 
$E_{b} \leq\left(m_{t}^{2}+m_{b}^{2}-m_{H^{+}}^{2}\right) /\left(2 m_{t}\right)$ and $0 \leq E_{g} \leq\left(m_{t}^{2}-\left(m_{b}+m_{H^{+}}\right)^{2}\right) /\left(2 m_{t}\right)$. Following ref. [28], it is convenient to introduce the scaled energy fractions $x_{i}=E_{i} / E_{b}^{\max }=2 E_{i} /\left(m_{t}(1+R-y)\right)$ ( $\mathrm{i}=\mathrm{b}, \mathrm{g}, \mathrm{B})$ where the scaled masses y and $\mathrm{R}$ are defined as $y=m_{H^{+}}^{2} / m_{t}^{2}$ and $R=m_{b}^{2} / m_{t}^{2}$. By neglecting the b-quark mass $m_{b}$, one has $x_{i}=2 E_{i} /\left(m_{t}(1-y)\right)$ so that $0 \leq\left(x_{b}, x_{g}\right) \leq 1$.

In the first step, we analyze the parton-level sector of the decay process (2.1) in the rest frame of a top quark. The angular distribution of the differential decay width $d \hat{\Gamma} / d x_{i}(i=$ $b, g$ ) of a polarized top quark is given by the following simple expression to clarify the correlation between the polarization of the top quark and its decay products

$$
\frac{d^{2} \hat{\Gamma}\left(t(\uparrow) \rightarrow b H^{+}(g)\right)}{d x_{i} d \cos \theta_{P}}=\frac{1}{2}\left\{\frac{d \hat{\Gamma}^{\text {unpol }}}{d x_{i}} \pm P \frac{d \hat{\Gamma}^{\text {pol }}}{d x_{i}} \cos \theta_{P}\right\}
$$

where $\mathrm{P}$ is the polarization degree of the top quark with $0 \leq P \leq 1$ so that $\mathrm{P}=1$ corresponds to $100 \%$ top quark polarization and $\mathrm{P}=0$ corresponds to an unpolarized top quark. In eq. (2.2) $d \hat{\Gamma}^{\mathrm{unpol}} / d x_{i}$ stands for the unpolarized differential rate, which is extensively calculated in $[23,24]$ up to NLO, and $d \hat{\Gamma}^{\text {pol }} / d x_{i}$ refers to the polarized one. The analytical expression for the differential partial width $d \hat{\Gamma}^{\mathrm{pol}} / d x_{i}$ depends on the selected helicity coordinate system. In the rest frame of a top quark decaying into a b-quark, a Higgs boson and a gluon, the final state particles define an event plane. Relative to this plane, we can define the polarization direction of the polarized top quark. For the decay process (2.1), there are two various choices of possible coordinate systems relative to the event plane where one differentiates between frames according to the orientation of the z-axis.

In [25], we calculated the angular distribution of the partial decay width $d \hat{\Gamma} / d x_{i}$ in a specific frame (system 1) where the three-momentum of the charged Higgs boson $\left(\vec{P}_{H^{+}}\right)$ pointed in the direction of the positive z-axis and the polar angle $\theta_{P}$ was defined as the angle between the polarization vector $\vec{P}_{t}$ of the top quark and the positive z-axis. The sign ' + ' in (2.2) stands for this system.

Here, we consider a different helicity coordinate system (system 2) where the threemomentum of the bottom quark points in the direction of the positive z-axis (see figure 1). In (2.2), the sign '-' stands for the system 2. The technical detail of our calculation will be presented in the next section. We will show that the results depend on the selected helicity system.

Having the parton-level differential decay rates $d \hat{\Gamma} / d x_{i}$, our main purpose is to evaluate the distribution in the scaled-energy $\left(x_{B}\right)$ of B-mesons in the polarized top quark rest frame. For this study, we evaluate the partial decay width of process (2.1) differential in $x_{B}, d \Gamma / d x_{B}$, at NLO where the normalized energy fraction of the B-meson is defined as $x_{B}=2 E_{B} /\left(m_{t}(1-y)\right)$. According to the factorization theorem of the QCD-improved parton model [34], the energy distribution of a B-meson can be expressed as the convolution of the parton-level spectrum with the nonperturbative fragmentation function $D_{i}^{B}\left(z, \mu_{F}\right)$, describing the hadronization $i \rightarrow B$,

$$
\frac{d \Gamma}{d x_{B}}=\sum_{i=b, g} \int_{x_{i}^{\min }}^{x_{i}^{\max }} \frac{d x_{i}}{x_{i}} \frac{d \hat{\Gamma}}{d x_{i}}\left(\mu_{R}, \mu_{F}\right) D_{i}^{B}\left(\frac{x_{B}}{x_{i}}, \mu_{F}\right)
$$




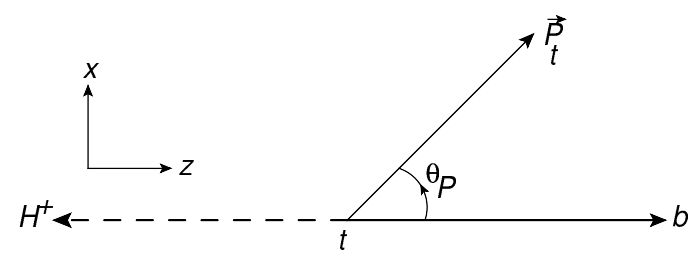

Figure 1. Polar angle $\theta_{P}$ defined in the second helicity coordinate system (system 2). $\vec{P}_{t}$ stands for the top polarization vector in the top rest frame.

where $d \hat{\Gamma} / d x_{i}(\mathrm{i}=\mathrm{b}, \mathrm{g})$ is the parton-level differential width of the process (2.1) in each selected helicity coordinate system. In the equation above, $\mu_{F}$ and $\mu_{R}$ are the factorization and the renormalization scales, respectively. In principle, one can use two different values for these scales; however, a choice often made consists of setting $\mu_{R}=\mu_{F}$ and we shall adopt this convention in our work. We will go back to the factorization theorem in section 4 , when our numerical analysis is presented.

In the next section, we present our analytic results for $d \hat{\Gamma} / d x_{i}(i=b, g)$ at NLO in the helicity system 2 .

\section{Analytic results for $d \hat{\Gamma} / d x_{i}$}

In this section we study the NLO radiative corrections to the partial decay width $t(\uparrow) \rightarrow b+H^{+}$in the general $2 \mathrm{HDM}$, where $H_{1}$ and $H_{2}$ are the doublets that their vacuum expectation values give masses to the down and up type quarks, respectively, and a linear combination of the charged components of $H_{1}$ and $H_{2}$ gives the physical charged Higgs $H^{ \pm}$. In general models with two Higgs doublets and generic coupling to all the quarks, it is difficult to avoid tree-level flavor-changing neutral currents. We, thus, limit ourselves to the models that naturally stop these problems by restricting the Higgs coupling to all quarks. Generally, there are four possibilities (called four models in the following) for the two Higgs doublets to couple to the fermions. In these models, the Yukawa couplings between the charged Higgs boson, the top and the bottom quarks are expressed as [1]

$$
L_{I}=\frac{g_{W}}{2 \sqrt{2} m_{W}} V_{t b} H^{+}\left[\bar{u}_{t}\left(p_{t}\right)\left\{A\left(1+\gamma_{5}\right)+B\left(1-\gamma_{5}\right)\right\} u_{b}\left(p_{b}\right)\right],
$$

where A and B are model-dependent parameters and $g_{W}$ is the weak coupling factor.

In the first model (model I) the doublet $H_{1}$ couples to all bosons and the doublet $\mathrm{H}_{2}$ couples to all the quarks. In this model, one has

$$
A=m_{t} \cot \beta, \quad B=-m_{b} \cot \beta .
$$

In the second model (model II), the doublet $H_{1}$ couples to the right-chiral down-type quarks and the doublet $H_{2}$ couples to the right-chiral up-type quarks. In this model, the interaction Lagrangian consists of

$$
A=m_{t} \cot \beta, \quad B=m_{b} \tan \beta
$$


Two other models are also possible (models III and IV) so that for the model III, both upand down-type quarks couple to the second doublet $\left(H_{2}\right)$ and all leptons to the first one while for the model IV, the roles of the two doublets are reversed with respect to Type II [35]. A summary of which type of fermions couple to $H_{1}$ and $H_{2}$ in models I-IV is given in table I of [35]. Here, we just mention that the charged Higgs boson contributions to processes which depend only on the quark sector (e.g., the process examined in (2.1)) are the same both in models I and IV and also in models II and III. Thus, the analytical results presented for the partonic process $t \rightarrow b H^{+}$in the models I and II can be also applied for models IV and III, respectively. Indeed, the difference between the models I and IV (also between the models II and III) is due to the leptonic sector of the decay process $t(\uparrow) \rightarrow b H^{+} \rightarrow b(B X)+H^{+}\left(\tau^{+} \nu_{\tau}\right)$, see table I of ref. [35].

In (3.2) and (3.3), $\tan \beta=\mathbf{v}_{2} / \mathbf{v}_{1}$ is the ratio of the vacuum expectation values of the two electrically neutral components of the two Higgs doublets. The models I and II are also known as type-I and type-II 2HDM scenarios.

\subsection{Born-level rate of $t \rightarrow b H^{+}$in ZM-VFNS}

The Born term amplitude in the 2HDM for the process $t(\uparrow) \rightarrow b+H^{+}$can either be expressed as a superposition of right- and left-chiral coupling factors, i.e. $M_{0}=\bar{u}_{b}\left\{g_{t}(1+\right.$ $\left.\left.\gamma_{5}\right) / 2+g_{b}\left(1-\gamma_{5}\right) / 2\right\} u_{t}$, or as a superposition of scalar and pseudoscaler coupling factors, i.e. $M_{0}=\bar{u}_{b}\left(a+b \gamma_{5}\right) u_{t}$, where $a=\left(g_{t}+g_{b}\right) / 2$ and $b=\left(g_{t}-g_{b}\right) / 2$. One also has $g_{b}=2 B$ and $g_{t}=2 A$ where $A$ and $B$ are defined in (3.2) and (3.3) for the models I and II. The inverse relation reads $\mathrm{a}=\mathrm{A}+\mathrm{B}$ and $\mathrm{b}=\mathrm{A}-\mathrm{B}$.

Therefore, for the Born amplitude squared one has: $\left|M_{0}\right|^{2}=2\left(p_{b} \cdot p_{t}\right)\left(a^{2}+b^{2}\right)+$ $2\left(a^{2}-b^{2}\right) m_{b} m_{t}+4 a b m_{t}\left(p_{b} \cdot s_{t}\right)$ where we replaced $\sum_{s_{t}} u\left(p_{t}, s_{t}\right) \bar{u}\left(p_{t}, s_{t}\right)=\left(\not p_{t}+m_{t}\right)$ in the unpolarized Dirac string by $u\left(p_{t}, s_{t}\right) \bar{u}\left(p_{t}, s_{t}\right)=\left(1-\gamma_{5} \phi_{t}\right)\left(\not p_{t}+m_{t}\right) / 2$ in the polarized state.

Considering figure 1, the polarization four-vector of the top quark in the top rest frame reads; $s_{t}=P\left(0 ; \sin \theta_{P} \cos \phi_{P}, \sin \theta_{P} \sin \phi_{P}, \cos \theta_{P}\right)$ and thus one has $p_{b} \cdot s_{t}=$ $-P\left(\left|\vec{p}_{b}\right| \cos \theta_{P}\right)$. This justifies the minus sign in eq. (2.2). Therefore, the tree-level helicity structure of differential rate reads

$$
\frac{d^{2} \hat{\Gamma}_{0}}{d x_{b} d \cos \theta_{P}}=\frac{1}{2}\left\{\hat{\Gamma}_{0}^{\text {unpol }}-P \hat{\Gamma}_{0}^{\text {pol }} \cos \theta_{P}\right\} \delta\left(1-x_{b}\right),
$$

where the unpolarized Born-level decay width is given by

$$
\hat{\Gamma}_{0}^{\text {unpol }}=\frac{m_{t}\left(a^{2}+b^{2}\right)}{16 \pi}(1+R-y) \times \lambda^{\frac{1}{2}}(1, R, y)\left\{1+\frac{2 \sqrt{R}}{1+R-y}\left(\frac{a^{2}-b^{2}}{a^{2}+b^{2}}\right)\right\},
$$

and the polarized tree-level one, reads

$$
\hat{\Gamma}_{0}^{\text {pol }}=\frac{m_{t}}{8 \pi} \lambda(1, R, y)(a b),
$$

where $\lambda(x, y, x)=x^{2}+y^{2}+z^{2}-2(x y+x z+y z)$ is the triangle function, $R=m_{b}^{2} / m_{t}^{2}$ and $y=m_{H^{+}}^{2} / m_{t}^{2}$. The above results are independent of the selected helicity frames and are in complete agreement with refs. [18-22]. 
In the limit of vanishing b-quark mass $(m \rightarrow 0 \equiv R \rightarrow 0)$ one has $\mathrm{a}=\mathrm{b}$ in the model I (or in the type-I 2HDM), then the tree-level decay width is simplified to

$$
\hat{\Gamma}_{0}^{\text {unpol }}=\hat{\Gamma}_{0}^{\text {pol }}=\frac{m_{t}^{3}}{8 \sqrt{2} \pi} G_{F}\left|V_{t b}\right|^{2}(1-y)^{2} \cot ^{2} \beta,
$$

and for the model II (type-II 2HDM), one has

$$
\hat{\Gamma}_{0}^{\text {unpol }}=\frac{m_{t}^{3}}{8 \sqrt{2} \pi} G_{F}\left|V_{t b}\right|^{2}(1-y)^{2}\left\{\cot ^{2} \beta+R \tan ^{2} \beta\right\} \times\left(1+\frac{4 R}{1-y}\left(\frac{1}{\cot ^{2} \beta+R \tan ^{2} \beta}\right)\right),
$$

and,

$$
\hat{\Gamma}_{0}^{\text {pol }}=\frac{m_{t}^{3}}{8 \sqrt{2} \pi} G_{F}\left|V_{t b}\right|^{2}(1-y)^{2}\left\{\cot ^{2} \beta-R \tan ^{2} \beta\right\}
$$

In (3.8) and (3.9), when the $R \tan ^{2} \beta$-term can be compared with $\cot ^{2} \beta$ therefore one cannot naively set $m_{b}=0$ in all expressions. For example, if we take $m_{b}=4.78 \mathrm{GeV}$, $m_{t}=172.98 \mathrm{GeV}, m_{H^{+}}=155 \mathrm{GeV}$ and $\tan \beta=4$ thus the second term in the curly brackets in (3.8) and (3.9) can become as large as $\mathcal{O}(20 \%)$ and this order will be larger when $\tan \beta$ is increased. Therefore, the $m_{b} \rightarrow 0$ approximation is not suitable for the type-II 2HDM. In this paper, we work in the type-I 2HDM and adopt, with a very good approximation, the Born term presented in (3.7) in the massless or zero-mass variable-flavor-number (ZMVFN) scheme [36] where the zero mass parton approximation is also applied to the bottom quark and the nonzero value of the b-quark mass only enter through the initial condition of the nonperturbative FF. The results for the type-II 2HDM, considering the massive or general-mass variable-flavor-number (GM-VFN) scheme will be presented in a forthcoming paper.

In the following, we present our analytical results for the $\mathcal{O}\left(\alpha_{s}\right)$ QCD corrections to the tree-level decay rate in the ZM-VFN scheme.

\subsection{Virtual corrections}

The QCD virtual one-loop corrections to the polarized differential width arise from emission and absorption of a virtual gluon from the same quark leg (quark self-energy) and from a virtual gluon exchanged between the top and bottom quark legs (vertex correction). In the ZM-VFN scheme all divergences including the infra-red (IR) and ultra-violet (UV) singularities which arise from the collinear- and the soft-gluon emissions, respectively, are regularized by dimensional regularization in $D=4-2 \epsilon$ space-time dimensions to become single poles in $\epsilon$. These singularities are subtracted at factorization scale $\mu_{F}$ and absorbed into the bare fragmentation functions (FFs) according to the modified minimal-subtraction scheme $(\overline{M S})$. The virtual contributions are the same in both helicity systems 1 and 2 , and more detail of our calculation can be found in [25]. We just mention that by neglecting the b-quark mass the counter term of the vertex consists of the top quark mass renormalization and the wave function renormalizations of both top and bottom quarks. Here, we just 
present our final result of the virtual corrections to the polarized differential decay rate as

$$
\frac{d \hat{\Gamma}^{\text {vir,pol }}}{d x_{b}}=\hat{\Gamma}_{0}^{\mathrm{pol}} \frac{\alpha_{s}\left(\mu_{R}\right)}{2 \pi} C_{F} \delta\left(1-x_{b}\right)\left(-\frac{1}{\epsilon^{2}}+\frac{F}{\epsilon}-\frac{F^{2}}{2}+\left(\frac{2}{y}-5\right) \ln (1-y)-2 \operatorname{Li}_{2}(y)-\frac{7}{8}-\frac{\pi^{2}}{12}\right),
$$

where, $F=2 \ln (1-y)-\ln \left(4 \pi \mu_{F}^{2} / m_{t}^{2}\right)+\gamma_{E}-5 / 2, C_{F}=\left(N_{c}^{2}-1\right) /\left(2 N_{c}\right)=4 / 3$ for $N_{c}=3$ quark colors, and $\operatorname{Li}_{2}(x)=-\int_{0}^{x}(d t / t) \ln (1-t)$ is the Spence function. Note that, all UVdivergences are canceled after summing all virtual corrections up but the IR-singularities are remaining which are labeled by $\epsilon$ in the above equation. Since the virtual corrections are the same in both helicity systems 1 and 2, the above result is in agreement with [18] where the authors have considered the first helicity system.

\subsection{Real gluon corrections}

In this section we calculate the $\mathcal{O}\left(\alpha_{s}\right)$ QCD corrections (i.e. $t(\uparrow) \rightarrow b H^{+} g$ ) which are needed to cancel the IR-singularities of the virtual corrections. In the rest frame of a top quark decaying into a Higgs boson, a bottom quark and a gluon the outgoing particles define an event plane so that relative to this plane one can define the spin direction of the polarized top quark. For our aim, two possible coordinate systems are defined as

$$
\begin{array}{rrrl}
\text { Systsem 1: } & \vec{p}_{H^{+}} \| \hat{z} ; & \left(\vec{p}_{b}\right)_{x} \geq 0 \\
\text { Systsem 2: } & \vec{p}_{b} \| \hat{z} ; & \left(\vec{p}_{H^{+}}\right)_{x} \geq 0 .
\end{array}
$$

The various helicity systems provide independent probes of light charged Higgs bosons in the polarized top quark decay dynamics.

In [25], we analyzed the spin-momentum correlation between the top quark polarization vector and the momenta of its decay products in the system 1 . In the present work, we study the same analysis in the system 2 and show that the energy spectrum of the outgoing B-meson depends on the helicity system selected. Considering the general form of angular distribution of the differential decay width (2.2), one has

$$
\frac{d^{2} \hat{\Gamma}^{\text {real }}}{d x_{b} d \cos \theta_{P}}=\frac{1}{2}\left(\frac{d \hat{\Gamma}^{\text {unpol,real }}}{d x_{b}}-P \frac{d \hat{\Gamma}^{\text {pol,real }}}{d x_{b}} \cos \theta_{P}\right)
$$

where $d \hat{\Gamma}^{\text {unpol }} / d x_{b}$ is presented in $[23,24]$.

The $\mathcal{O}\left(\alpha_{s}\right)$ real gluon (tree-graph) contribution to the differential decay rate results from the square of the real amplitude as $\left|M^{\text {real }}\right|^{2}=M^{\text {real } \dagger} \cdot M^{\text {real }}$, where $M^{\text {real }}$ reads

$$
M^{\text {real }}=g_{s} \frac{\lambda^{a}}{2} \bar{u}\left(p_{b}, s_{b}\right)\left\{\frac{2 p_{t}^{\mu}-\not p_{g} \gamma^{\mu}}{2 p_{t} \cdot p_{g}}-\frac{2 p_{b}^{\mu}+\gamma^{\mu} \not p_{g}}{2 p_{b} \cdot p_{g}}\right\}\left(a \mathbf{1}+b \gamma_{5}\right) u\left(p_{t}, s_{t}\right) \epsilon_{\mu}^{\star}\left(p_{g}, r\right),
$$

where the polarization vector of the real gluon with the momentum $p_{g}$ and spin $\mathrm{r}$ is denoted by $\epsilon\left(p_{g}, r\right)$. The first and second terms in the curly brackets refer to the real gluon emission from the top and the bottom quarks, respectively. 
As before, to regulate the IR-divergences we work in $D=4-2 \epsilon$ dimensions, therefore from the definition of decay rate, one has

$$
d \hat{\Gamma}^{\text {real }}=\frac{\mu_{F}^{2(4-D)}}{2 m_{t}}\left|M^{\text {real }}\right|^{2} d P S\left(p_{t}, p_{b}, p_{g}, p_{H^{+}}\right),
$$

where, the Phase Space element reads

$$
d P S=\frac{d^{D-1} \vec{p}_{b}}{(2 \pi)^{D-1} 2 E_{b}} \frac{d^{D-1} \vec{p}_{H^{+}}}{(2 \pi)^{D-1} 2 E_{H^{+}}} \frac{d^{D-1} \vec{p}_{g}}{(2 \pi)^{D-1} 2 E_{g}} \times(2 \pi)^{D} \delta^{D}\left(p_{t}-p_{b}-p_{H^{+}}-p_{g}\right) .
$$

To calculate the real polarized differential decay rate $d \hat{\Gamma}^{\text {pol,real }} / d x_{b}$, we fix the momentum of the bottom quark in eq. (3.14) and integrate over the gluon energy which ranges as $m_{t} S\left(1-x_{b}\right) \leq E_{g} \leq m_{t} S\left(1-x_{b}\right) /\left(1-2 S x_{b}\right)$ where $S=(1-y) / 2$. Also, to get the correct finite terms one has to normalize it to the Born width (3.7) which is evaluated in the dimensional regularization at $\mathcal{O}\left(\epsilon^{2}\right)$, i.e. $\hat{\Gamma}_{0}^{\text {pol }} \rightarrow \hat{\Gamma}_{0}^{\text {pol }}\left\{1-\epsilon\left(\gamma_{E}+2 \ln S-\ln \left(4 \pi \mu_{F}^{2} / m_{t}^{2}\right)\right)\right\}$.

Thus, in the second helicity coordinate system the contribution of the real gluon emission into the normalized differential decay width is given by

$$
\begin{aligned}
\frac{1}{\hat{\Gamma}_{0}^{\text {pol }}} \frac{d \hat{\Gamma}^{\text {real }, \text { pol }}}{d x_{b}}= & \frac{\alpha_{s}}{2 \pi} C_{F}\left\{\delta ( 1 - x _ { b } ) \left[\frac{1}{\epsilon^{2}}-\frac{1}{\epsilon}\left(F+\frac{3}{2}\right)+\frac{F^{2}}{2}\right.\right. \\
& \left.+\frac{3}{2} F-2 \frac{y}{1-y} \ln y+2 \operatorname{Li}_{2}(1-y)-\frac{\pi^{2}}{4}+\frac{5}{8}\right] \\
& \left.+\frac{1+x_{b}^{2}}{\left(1-x_{b}\right)_{+}}\left[-\frac{1}{\epsilon}+2 \ln x_{b}+F+\frac{3}{2}\right]+2\left(1+x_{b}^{2}\right)\left(\frac{\ln \left(1-x_{b}\right)}{1-x_{b}}\right)_{+}\right\}
\end{aligned}
$$

where $F=2 \ln (1-y)-\ln \left(4 \pi \mu_{F}^{2} / m_{t}^{2}\right)+\gamma_{E}-5 / 2$ and the plus distributions are defined as usual.

\subsection{Analytic results for partial decay rates $d \hat{\Gamma} / d x_{i}$ in the helicity system 2}

The NLO expression for the $d \hat{\Gamma}^{\text {pol }} / d x_{b}$ is obtained by summing the Born term, the virtual one-loop and the real gluon contributions. Our result for the helicity coordinate system 2 , is as follows

$$
\begin{aligned}
\frac{d \hat{\Gamma}^{\text {pol }}}{d x_{b}}= & \hat{\Gamma}_{0}^{\text {pol }}\left\{\delta\left(1-x_{b}\right)+\frac{\alpha_{s}\left(\mu_{R}\right)}{2 \pi}\left\{\left[-\frac{1}{\epsilon}+\gamma_{E}-\ln 4 \pi\right] \times P_{q q}^{(0)}\left(x_{b}\right)\right.\right. \\
& +C_{F}\left[\delta ( 1 - x _ { b } ) \left[2 \frac{1-y}{y} \ln (1-y)-4-\frac{2 y}{1-y} \ln y-\frac{\pi^{2}}{3}\right.\right. \\
& \left.-2 \operatorname{Li}_{2}(y)+2 \operatorname{Li}_{2}(1-y)-\frac{3}{2} \ln \frac{\mu_{F}^{2}}{m_{t}^{2}}\right]-\frac{1+x_{b}^{2}}{\left(1-x_{b}\right)_{+}}\left[1-2 \ln \left(x_{b}(1-y)\right)+\ln \frac{\mu_{F}^{2}}{m_{t}^{2}}\right] \\
& \left.\left.\left.+2\left(1+x_{b}^{2}\right)\left(\frac{\ln \left(1-x_{b}\right)}{1-x_{b}}\right)_{+}\right]\right\}\right\}
\end{aligned}
$$


where $P_{q q}^{(0)}$ is the time-like $q \rightarrow q$ splitting function at leading order [37-39], so

$$
P_{q q}^{(0)}\left(x_{b}\right)=C_{F}\left(\frac{1+x_{b}^{2}}{\left(1-x_{b}\right)_{+}}+\frac{3}{2} \delta\left(1-x_{b}\right)\right)
$$

Since, the bottom-flavored hadrons can be also produced through the hadronization of the emitted real gluon at NLO, we also need the differential decay rate $d \hat{\Gamma}^{\text {pol }} / d x_{g}$ in the ZMVFN scheme. To calculate the $d \hat{\Gamma}^{\text {pol }} / d x_{g}$ we start form eq. (3.14) and fix the momentum of the gluon and integrate over the bottom quark energy so that $m_{t} S\left(1-x_{g}\right) \leq E_{b} \leq$ $m_{t} S\left(1-x_{g}\right) /\left(1-2 S x_{g}\right)$. Since we fix the gluon momentum, then there will be no soft singularities in the $d \hat{\Gamma}^{\text {pol }} / d x_{g}$. The result in the helicity system 2 , reads

$$
\begin{aligned}
\frac{d \hat{\Gamma}^{\mathrm{pol}}}{d x_{g}}= & \hat{\Gamma}_{0}^{\mathrm{pol}}\left\{\frac { \alpha _ { s } ( \mu _ { R } ) } { 2 \pi } \left\{\left[-\frac{1}{\epsilon}+\gamma_{E}-\ln 4 \pi\right] \times P_{g q}^{(0)}\left(x_{g}\right)\right.\right. \\
& +C_{F}\left[3-\frac{y^{2}}{4 S\left(1-2 S x_{g}\right)^{2}}+\frac{1}{S x_{g}^{2}} \ln \left(1-2 S x_{g}\right)\right. \\
& \left.\left.\left.+\frac{12 S^{2}-8 S+1}{4 S\left(1-2 S x_{g}\right)}-\frac{x_{g}}{2}-\frac{1+\left(1-x_{g}\right)^{2}}{x_{g}}\left(\ln \frac{\mu_{F}^{2}}{m_{t}^{2}}-\ln \frac{4 S^{2} x_{g}^{2}\left(1-x_{g}\right)^{2}}{1-2 S x_{g}}\right)\right]\right\}\right\}
\end{aligned}
$$

where $P_{g q}^{(0)}$ is the time-like $q \rightarrow g$ splitting function at LO [37-39],

$$
P_{g q}^{(0)}\left(x_{g}\right)=C_{F}\left(\frac{1+\left(1-x_{g}\right)^{2}}{x_{g}}\right) .
$$

To subtract the collinear singularities remaining in eqs. (3.17) and (3.19), we apply the modified minimal subtraction $(\overline{\mathrm{MS}})$ scheme where the collinear singularities are absorbed into the bare FFs. This renormalizes the FFs and generates the finite terms of the form $\alpha_{s} \ln \left(m_{t}^{2} / \mu_{F}^{2}\right)$ in the polarized differential decay rates. According to this scheme, in order to get the $\overline{\mathrm{MS}}$ coefficient functions we shall have to subtract from eqs. (3.17) and (3.19) the $\mathcal{O}\left(\alpha_{s}\right)$ term multiplying the characteristic $\overline{M S}$ constant $\left(-1 / \epsilon+\gamma_{E}-\ln 4 \pi\right)$. In this work we set $\mu_{R}=\mu_{F}=m_{t}$, so that in eqs. (3.17) and (3.19) the terms proportional to $\ln \left(m_{t}^{2} / \mu_{F}^{2}\right)$ vanish.

Integrating $d \hat{\Gamma}^{\mathrm{pol}} / d x_{b}$ of eq. (3.17) over $x_{b}\left(0<x_{b}<1\right)$, we obtain the NLO renormalized decay rate as

$$
\hat{\Gamma}^{\mathrm{pol}}=\hat{\Gamma}_{0}^{\mathrm{pol}}\left\{1-\frac{C_{F} \alpha_{s}}{2 \pi}\left[\frac{2 y}{1-y} \ln y+\left(5-\frac{2}{y}\right) \ln (1-y)+2 \mathrm{Li}_{2}(y)-2 \mathrm{Li}_{2}(1-y)-\frac{7}{2}+\pi^{2}\right]\right\}
$$

Our previous result for $\hat{\Gamma}^{\mathrm{pol}}\left(=\int_{0}^{1} d x_{b} d \hat{\Gamma}^{\mathrm{pol}} / d x_{b}\right)$ in the helicity system 1 [25] was in complete agreement with ref. [18], but the above result computed in the second frame (system 2) is completely new. 


\section{Numerical analysis in type-I 2HDM}

In the 2HDM, the mass of charged Higgs bosons is restricted by $m_{H^{ \pm}}>m_{W^{ \pm}}$at treelevel [40], but this restriction does not hold for some regions of parameter space after including radiative corrections. In this model, $m_{H^{ \pm}}$is strongly correlated with the mass of other Higgs bosons. In [19], it is mentioned that a charged Higgs boson with a mass range $80 \mathrm{GeV} \leq m_{H^{ \pm}} \leq 160 \mathrm{GeV}$ is a logical possibility and its effects should be searched for in the decay mode $t \rightarrow b H^{+} \rightarrow B \tau^{+} \nu_{\tau}+X$. On the other hand, the recent results of a search for evidence of a charged Higgs boson in $19.5-19.7 \mathrm{fb}^{-1}$ of proton-proton collision data recorded at $\sqrt{s}=8 \mathrm{TeV}$ are reported by the CMS $[14,15]$ and the ATLAS $[16,17]$ collaborations, using the $\tau+$ jets channel with a hadronically decaying $\tau$ lepton in the final state. According to figure 7 of refs. $[16,17]$, the large region in the $m_{H^{+}}-\tan \beta$ parameter space is excluded for $m_{H^{+}}=80-160 \mathrm{GeV}$. So, the unexcluded regions of this parameter space include the charged Higgs masses as $90 \leq m_{H^{+}} \leq 100 \mathrm{GeV}$ (with $6<\tan \beta<10$ ) and $140 \leq m_{H^{+}} \leq 160 \mathrm{GeV}$ (with $3<\tan \beta<21$ ). See also figure 9 of refs. [14, 15]. Therefore, these values of $m_{H^{ \pm}}$and $\tan \beta$ are still allowed and in this work our prediction and analysis is restricted to these regions. However, a definitive search of the charged Higgs bosons over this part of the $m_{H^{+}}-\tan \beta$ parameter space still has to be carried out by the LHC experiments.

Here, for our numerical analysis we adopt the input parameter values from ref. [40] as; $G_{F}=1.16637 \times 10^{-5} \mathrm{GeV}^{-2}, m_{t}=172.98 \mathrm{GeV}, m_{b}=4.78 \mathrm{GeV}, m_{W}=80.399 \mathrm{GeV}, m_{B}=$ $5.279 \mathrm{GeV}$, and $\left|V_{t b}\right|=0.999152$. Considering the unexcluded $m_{H^{+}}-\tan \beta$ parameter space from the ATLAS experiments $[16,17]$, we also consider $m_{H^{+}}=95,155 \mathrm{GeV}$ and $160 \mathrm{GeV}$.

In the ZM-VFN scheme the polarized and unpolarized decay rates at the Born level are the same in both helicity systems (see (3.7) and corresponding explanation) and from now we label them by $\hat{\Gamma}_{0}\left(=\hat{\Gamma}_{0}^{\text {unpol }}=\hat{\Gamma}_{1,0}^{\text {pol }}=\hat{\Gamma}_{2,0}^{\text {pol }}\right)$. Our result for the unpolarized rate at NLO, is

$$
\begin{array}{lll}
\hat{\Gamma}^{\text {unpol }}=\hat{\Gamma}_{0}(1-0.010), & \text { for } & m_{H^{+}}=160 \mathrm{GeV} \\
\hat{\Gamma}^{\text {unpol }}=\hat{\Gamma}_{0}^{\prime}(1-0.028), & \text { for } & m_{H^{+}}=155 \mathrm{GeV} \\
\hat{\Gamma}^{\text {unpol }}=\hat{\Gamma}_{0}^{\prime \prime}(1-0.086), & \text { for } & m_{H^{+}}=95 \mathrm{GeV}
\end{array}
$$

and for the polarized rate in the system 1 , one has

$$
\begin{array}{lll}
\hat{\Gamma}_{1}^{\text {pol }}=\hat{\Gamma}_{0}(1-0.037), & \text { for } & m_{H^{+}}=160 \mathrm{GeV} \\
\hat{\Gamma}_{1}^{\text {pol }}=\hat{\Gamma}_{0}^{\prime}(1-0.038), & \text { for } \quad & m_{H^{+}}=155 \mathrm{GeV} \\
\hat{\Gamma}_{1}^{\text {pol }}=\hat{\Gamma}_{0}^{\prime \prime}(1-0.043), & \text { for } & m_{H^{+}}=95 \mathrm{GeV}
\end{array}
$$

and for the NLO polarized width in the helicity system 2, we have

$$
\begin{array}{lll}
\hat{\Gamma}_{2}^{\text {pol }}=\hat{\Gamma}_{0}(1-0.033), & \text { for } & m_{H^{+}}=160 \mathrm{GeV} \\
\hat{\Gamma}_{2}^{\text {pol }}=\hat{\Gamma}_{0}^{\prime}(1-0.051), & \text { for } \quad m_{H^{+}}=155 \mathrm{GeV} \\
\hat{\Gamma}_{2}^{\text {pol }}=\hat{\Gamma}_{0}^{\prime \prime}(1-0.109), & \text { for } \quad & m_{H^{+}}=95 \mathrm{GeV} .
\end{array}
$$

In the above results the $\hat{\Gamma}_{0}, \hat{\Gamma}_{0}^{\prime}$ and $\hat{\Gamma}_{0}^{\prime \prime}$ depend on the $m_{H^{+}}$and $\tan \beta$, see (3.7). As is seen, the NLO polarized decay rates depend on the selected helicity coordinate system. In 
obtaining the results above, we applied the unpolarized decay rate presented in [23, 24] and the polarized ones in the first and second helicity systems given in [25] and (3.21), respectively.

Here, we take a moment to mention to an important effect which was neglected in our previous calculations of charged Higgs production. Indeed, there are some interference effects between the on-shell top pair production and the off-shell production through the gluon splitting. The latter is mostly important for charged Higgs masses well above the top quark mass (typically $m_{H^{ \pm}} \gtrsim 200 \mathrm{GeV}$ ) where the top quark decay is kinematically forbidden and charged Higgs production can only occur when the off-shell top quark possibly is considered. For the intermediate-mass range $\left(145 \lesssim m_{H^{ \pm}} \lesssim 200 \mathrm{GeV}\right)$, the interplay effects between top quark resonant and non-resonant diagrams can not be neglected. The effect is, however, at the level of $10 \%$ (depending on the specific values of $\tan \beta$ and $m_{H^{ \pm}}$) and decreases with lower charged Higgs masses. More detail along with a convenient discussion can be found in [41].

After our numerical analysis of decay widths we are now in a situation to present our phenomenological predictions for the scaled-energy $\left(x_{B}\right)$ spectrum of bottom-flavored mesons (B) inclusively produced in polarized top decays in the type-I 2HDM. To show our predictions for the $x_{B}$-distribution, ignoring the interference effect with a good accuracy, we consider the doubly differential distribution $d^{2} \Gamma /\left(d x_{B} d \cos \theta_{P}\right)$ of the partial width of the decay $t(\uparrow) \rightarrow B H^{+}+X$ in the system 2. Here, $x_{B}=2 E_{B} /\left(m_{t}(1-y)\right)$ is the scaledenergy fraction of the B-meson in the top quark rest frame, where the energy of B-meson ranges from $E_{B}^{\min }=m_{B}$ to $E_{B}^{\max }=\left(m_{t}^{2}+m_{B}^{2}-m_{H}^{2}\right) /\left(2 m_{t}\right)$.

According to the factorization formula (2.3), the required ingredients for this study are the parton-level differential decay widths (3.17) and (3.19) and the fragmentation functions (FFs) $D_{b}^{B}(z)$ and $D_{g}^{B}(z)$ which describe the splitting of $b \rightarrow B$ and $g \rightarrow B$, respectively. To describe these hadronization processes, from ref. [42] we employ the nonperturbative B-hadron FFs determined at NLO in the ZM-VFN scheme through a global fit to $e^{+} e^{-}$ annihilation data taken by OPAL [43], ALEPH [44] and SLD [45, 46]. In ref. [42] authors used a simple power model $D_{b}\left(z, \mu_{F}^{\text {ini }}\right)=N z^{\alpha}(1-z)^{\beta}$ as the initial condition for the $b \rightarrow B \mathrm{FF}$ at $\mu_{F}^{\mathrm{ini}}=4.5 \mathrm{GeV}$, while the gluon and light-quark FFs were generated via the DGLAP evolution equations [37-39]. The fit yielded the values $N=4684.1, \alpha=16.87$, and $\beta=2.628$ for the FF parameters.

Considering the unexcluded $2 \mathrm{HDM} m_{H^{+}}-\tan \beta$ parameter space from the CMS $[14,15]$ and the ATLAS $[16,17]$ experiments, in figure 2 we show our prediction for the $x_{B}$-spectrum at NLO in the system 2 , taking $m_{H^{+}}=95 \mathrm{GeV}$ (solid line), $m_{H^{+}}=155 \mathrm{GeV}$ (dotted line) and $m_{H^{+}}=160 \mathrm{GeV}$ (dot-dashed line) where $\tan \beta=8$ is fixed for all predictions. As is seen, when $m_{H^{+}}$increases the size of decay rate decreases but the peak position is shifted towards higher values of $x_{B}$.

Considering the results of the CMS $[14,15]$ and ATLAS $[16,17]$ experiments where $4 \leq \tan \beta \leq 16$ is allowed for $m_{H^{+}}=155 \mathrm{GeV}$, in figure 3 we study the energy spectrum of B-meson in the helicity system 2 for different values of $\tan \beta=8$ (solid line), 12 (dashed line) and 16 (dot-dashed line), where the mass of Higgs boson is set to $m_{H^{+}}=155 \mathrm{GeV}$ for all analysis. As is seen, when $\tan \beta$ increases the size of decay rate decreases. This is obvious because $\hat{\Gamma}_{0}(3.7)$ is proportional to $\cot ^{2} \beta$. 


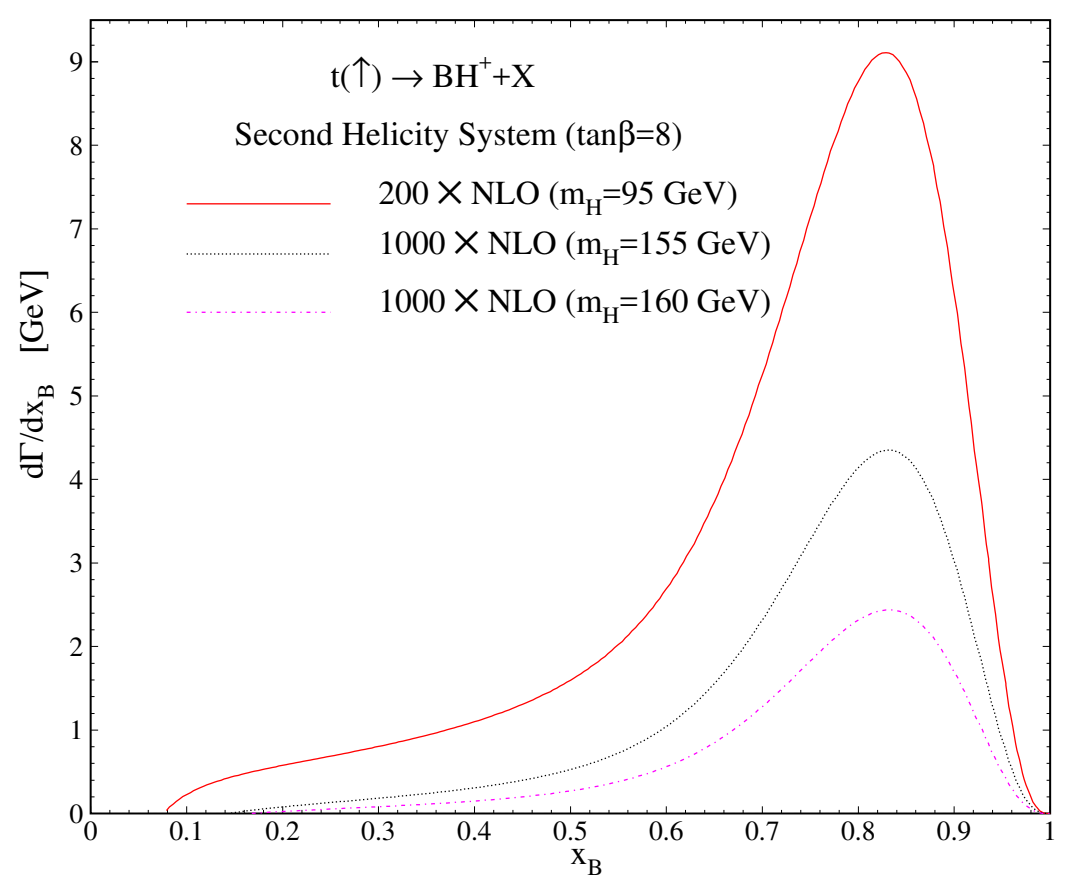

Figure 2. The NLO $x_{B}$-spectrum $\left(d \Gamma / d x_{B}\right)$ in polarized top decay in the helicity coordinate system 2 with $\tan \beta=8$ and $m_{H^{+}}=95 \mathrm{GeV}$ (solid line), $m_{H^{+}}=155$ (dotted line) and $160 \mathrm{GeV}$ (dot-dashed line).

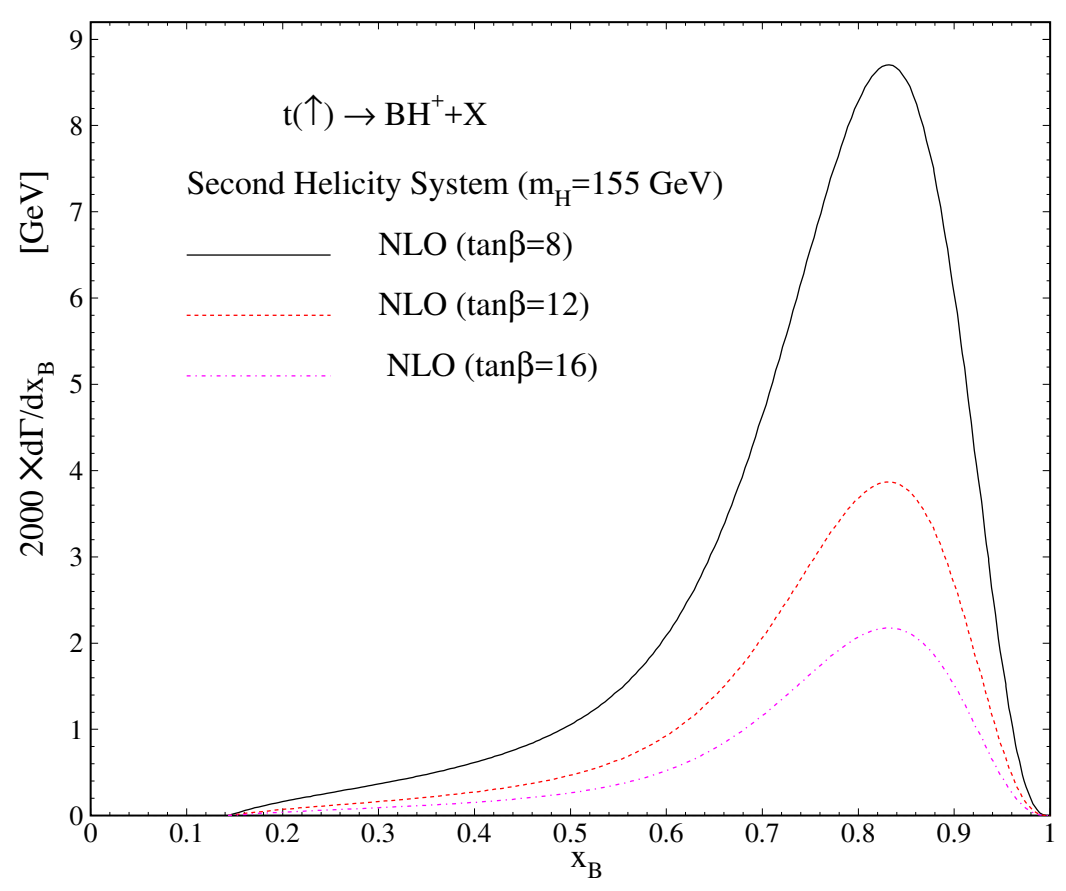

Figure 3. $x_{B}$ spectrum in polarized top decay in the type-I $2 \mathrm{HDM}$ with different values of $\tan \beta=8$, 12 and 16. The charged Higgs boson mass is set to $m_{H^{+}}=155 \mathrm{GeV}$. The analysis is done in the second helicity coordinate system. 


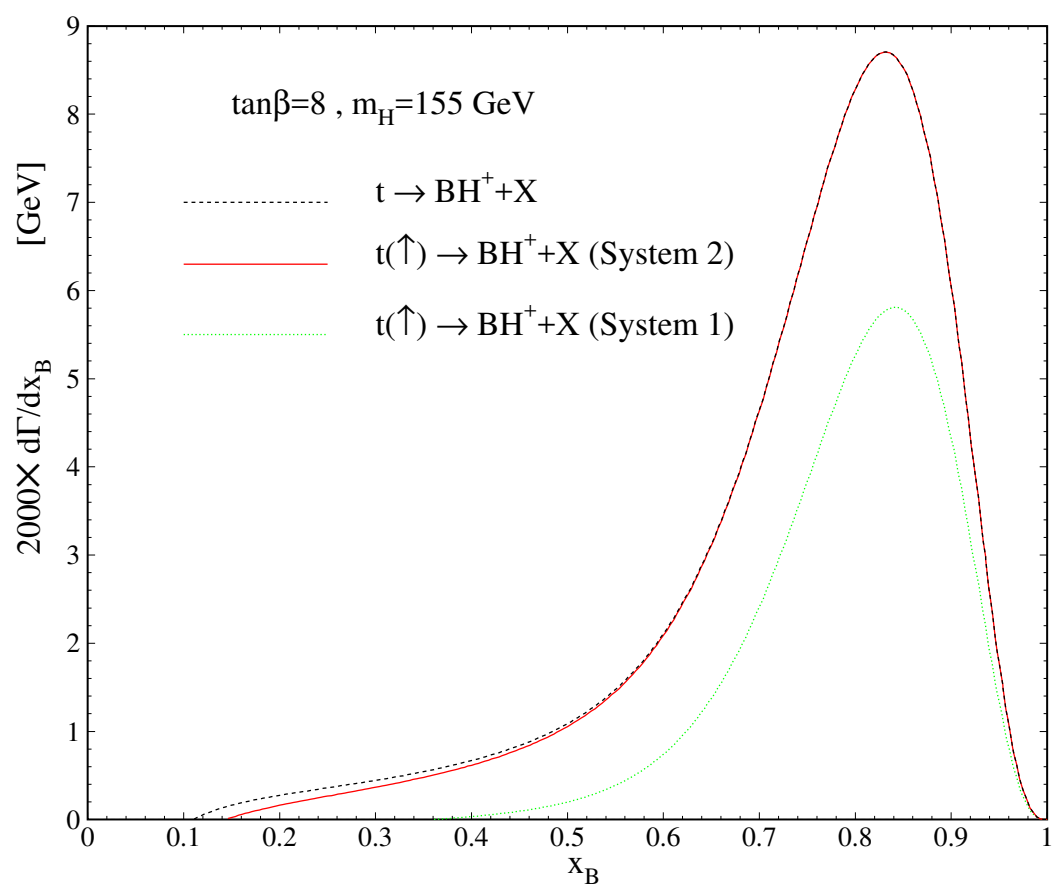

Figure 4. $d \Gamma / x_{B}$ as a function of $x_{B}$ in the type-I 2HDM considering the ZM-VFN scheme. The unpolarized (dashed line) and polarized partial decay rates are compared at NLO taking $m_{H^{+}}=155 \mathrm{GeV}, \tan \beta=8$ and $\mu_{R}=\mu_{F}=m_{t}$. For the polarized top decays we used the helicity system 1 (dotted line) and 2 (solid line). Detail are discussed in the text.

In figure 4 , taking $m_{H^{+}}=155 \mathrm{GeV}$ and $\tan \beta=8$ the NLO 2HDM energy spectrum of B-mesons from polarized top decays, $t(\uparrow) \rightarrow B H^{+}+X$, in the first (dotted line) and second (solid line) helicity coordinate systems are shown. As is seen the energy distributions obtained from our analysis in two various systems are different and the size of NLO correction is larger in the system 2. For more comparison, we also plotted the energy distribution of B-mesons through unpolarized top quark decays (dashed line). A considerable point is that the size of NLO corrections is the same both for the polarized top decay in the helicity system 2 and for the unpolarized one, except for small values of $x_{B}\left(0.14<x_{B}<0.45\right)$. In this region the unpolarized distribution is larger.

In figure 5 , as in figure 4, the same comparisons are done but for $m_{H^{+}}=95 \mathrm{GeV}$. Our results show that in these cases the NLO corrections are similar in the shape, however, the unpolarized distribution shows a more enhancement in size at NLO.

To obtain the energy spectrum of B-meson we used the factorization formula (2.3) where $\mu_{F}$ and $\mu_{R}$ are the factorization and the renormalization scales, respectively. In principle, two different values for these scales can be selected, however, we adopted $\mu_{R}=$ $\mu_{F}=m_{t}$ in our calculations. In figure 6 , we studied the dependence of the B-meson spectrum on these scales considering three different values: $\mu=m_{t} / 2$ (dots), $\mu=m_{t}$ (solid) and $\mu=2 m_{t}$ (dashes). This can be considered as a theoretical uncertainty due to scale variation.

It should be pointed out that our formalism elaborated here can be also extended to the production of hadron species other than bottom-flavored hadrons, such as pions, kaons 


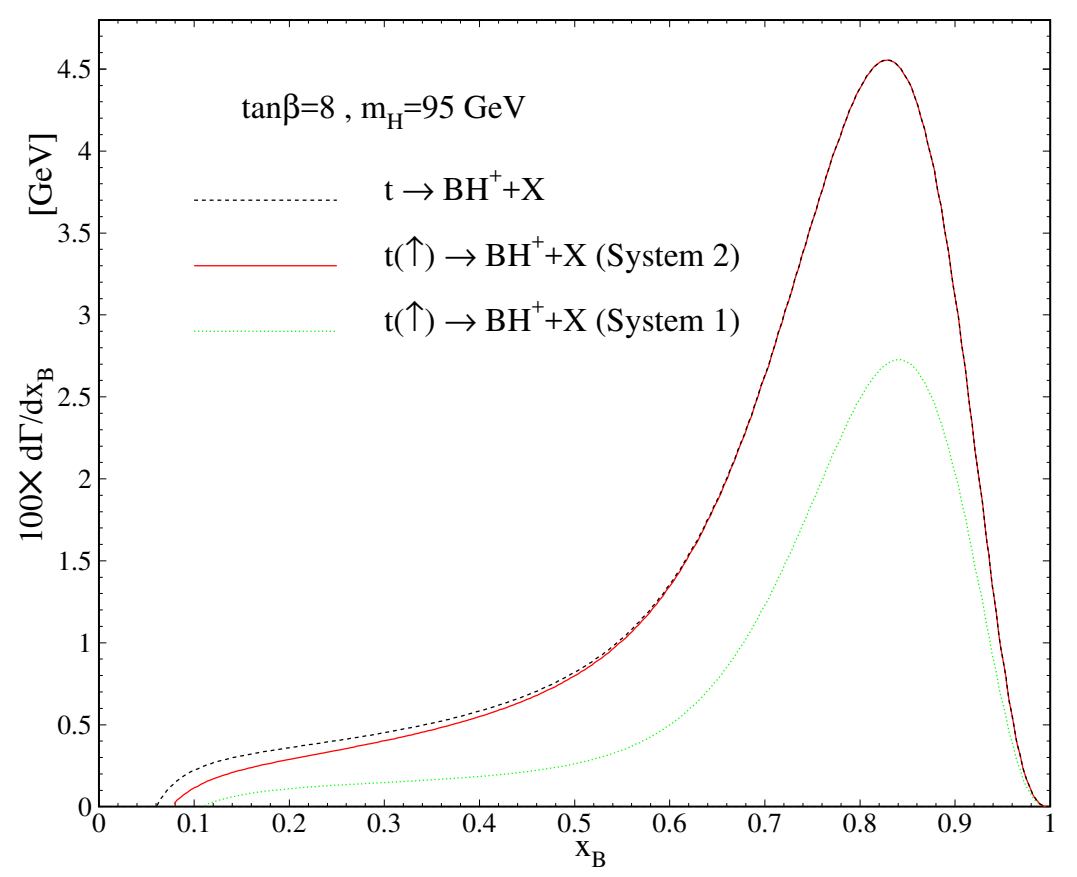

Figure 5. As in figure 4, but for $m_{H^{+}}=95 \mathrm{GeV}$. This mass is not excluded by the ATLAS experiments $[16,17]$.

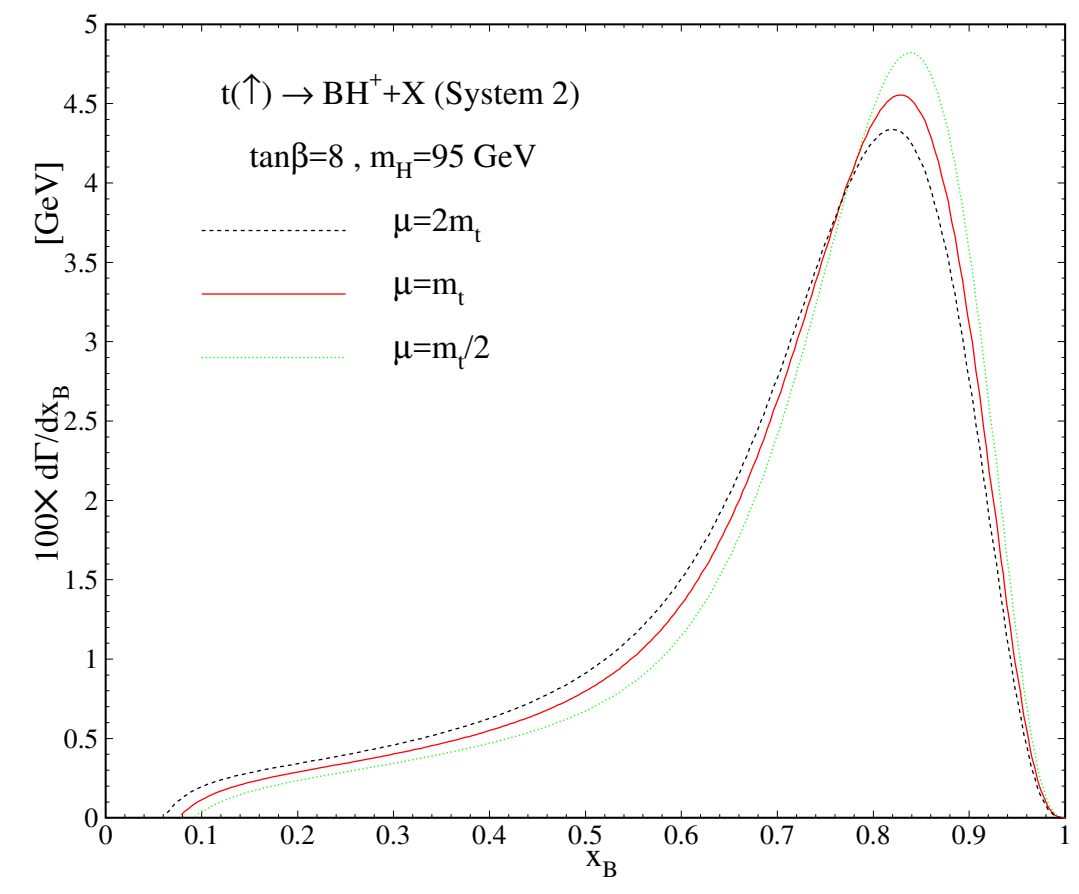

Figure 6. $x_{B}$ spectrum in polarized top decay for three different scales: $\mu=m_{t} / 2$ (dots), $\mu=m_{t}$ (solid) and $\mu=2 m_{t}$ (dashes). 
and protons, etc., using the nonperturbative $(b, g) \rightarrow \pi / K / P$ FFs extracted in our recent works [47-50], relying on their universality and scaling violations [51].

\section{Conclusions}

Charged Higgs bosons $\left(H^{ \pm}\right)$are predicted in models consisting of at least two Higgs doublets, of which the simplest are the two-Higgs-doublet models (2HDM). The chargedHiggses have been searched for in high energy experiments, in particular, at the Tevatron, ATLAS and CMS but they have not been seen so far. The discovery of a charged Higgs would represent unambiguous evidence for the presence of physics beyond the SM. There are many reasons, both from theoretical considerations and experimental observations, to except physics beyond the SM, such as the hierarchy problem, neutrino masses and dark matter.

In the 2HDM, the main production mode of light charged Higgs boson $\left(m_{H^{+}}<m_{t}\right)$ is through the top quark decay, $t \rightarrow b H^{+}$. On the other hand, bottom quarks hadronize, via $b \rightarrow B+X$, before they decay, so that the decay process $t \rightarrow B H^{+}+X$ is of prime importance at the LHC. Therefore, the study of scaled-energy $\left(x_{B}\right)$ distribution of the bottom-flavored mesons (B) inclusively produced in top quark decays is proposed as a new channel to indirect search for the light charged Higgs bosons. For this study, we need to evaluate the quantity $d \Gamma / d x_{B}$.

In $[23,24]$, we studied the energy spectrum of the B-mesons in unpolarized top decays into a charged-Higgs boson and a b-quark at NLO in the 2HDM. In [25] we studied the spin-dependent energy distribution of B-mesons produced through the polarized top decays at NLO in a special helicity coordinate system (system 1), where the event plane lied in the $(\mathrm{x}, \mathrm{z})$ plane and the Higgs three-momentum was along the $\mathrm{z}$-axis. In the present work, we have presented results on the NLO radiative corrections to the spin-dependent differential width $d^{2} \Gamma /\left(d x_{B} d \cos \theta_{P}\right)$, applying a different helicity system (system 2) where the z-axis is defined by the b-quark 3-momentum. This provides an independent probe of charged Higgses and also any deviation of the B-meson energy spectrum from the SM predictions can be considered as a signal for the existence of charged Higgs at the LHC. To make these predictions we obtained the analytical results for the parton-level differential decay width $d \hat{\Gamma}\left(t(\uparrow) \rightarrow b H^{+}(+g)\right) / d x_{a}(\mathrm{a}=\mathrm{b}, \mathrm{g})$ in two helicity systems 1 and 2 . Our result for the unpolarized differential decay width $d \hat{\Gamma}\left(t \rightarrow b H^{+}\right) / d x_{b}$ was in complete agreement with refs. [19-22] after integration over $0 \leq x_{b} \leq 1$, and our result for the polarized one in the system 1 was in agreement with [18] after integration over $x_{b}$. Here, using the same techniques we calculated the polarized differential width in the helicity system 2 and we also computed, for the first time, the polarized rate in the system 2 . We found that the polarized results depend on the selected helicity system, extremely.

For our numerical analysis, considering the recent results reported by the CMS $[14,15]$ and ATLAS $[16,17]$ collaborations we restricted ourselves to the unexcluded regions of the $2 \mathrm{HDM} m_{H^{+}}-\tan \beta$ parameter space which include $90 \leq m_{H^{+}} \leq 100 \mathrm{GeV}$ (with $6<\tan \beta<10$ ) and $140 \leq m_{H^{+}} \leq 160 \mathrm{GeV}$ (with $3<\tan \beta<21$ ). 
Since, highly polarized top quarks will become available at hadron colliders through single top production processes, which occur at the $33 \%$ level of the $t \bar{t}$ pair production rate [52], and in top quark pairs produced in future linear $e^{+} e^{-}$-colliders [53-55] these studies can be considered as a new channel to search for the charged Higss bosons.

\section{Acknowledgments}

We would like to thank Rebeca Gonzalez Suarez from the LHC top working group for reading the manuscript and also for importance discussion and comments. We warmly acknowledge the CERN TH-PH division for its hospitality where a portion of this work was performed.

Open Access. This article is distributed under the terms of the Creative Commons Attribution License (CC-BY 4.0), which permits any use, distribution and reproduction in any medium, provided the original author(s) and source are credited.

\section{References}

[1] J.F. Gunion, H. Haber, G. Kane and S. Dawson, The Higgs Hunter's Guide, Addison-Wesley, Reading Massachusetts U.S.A. (1990).

[2] T.P. Cheng and L.-F. Li, Neutrino Masses, Mixings and Oscillations in $\mathrm{SU}(2) \times \mathrm{U}(1)$ Models of Electroweak Interactions, Phys. Rev. D 22 (1980) 2860 [INSPIRE].

[3] T.D. Lee, A Theory of Spontaneous T Violation, Phys. Rev. D 8 (1973) 1226 [INSPIRE].

[4] A. Djouadi, The Anatomy of electro-weak symmetry breaking. II. The Higgs bosons in the minimal supersymmetric model, Phys. Rept. 459 (2008) 1 [hep-ph/0503173] [INSPIRE].

[5] K. Inoue, A. Kakuto, H. Komatsu and S. Takeshita, Aspects of Grand Unified Models with Softly Broken Supersymmetry, Prog. Theor. Phys. 68 (1982) 927 [Erratum ibid. 70 (1983) 330] [INSPIRE].

[6] U. Langenfeld, S. Moch and P. Uwer, New results for $t \bar{t}$ production at hadron colliders, arXiv:0907.2527 [INSPIRE].

[7] S. Moch and P. Uwer, Theoretical status and prospects for top-quark pair production at hadron colliders, Phys. Rev. D 78 (2008) 034003 [arXiv:0804.1476] [INSPIRE].

[8] N. Kidonakis and R. Vogt, The Theoretical top quark cross section at the Tevatron and the LHC, Phys. Rev. D 78 (2008) 074005 [arXiv:0805.3844] [INSPIRE].

[9] M. Aoki, R. Guedes, S. Kanemura, S. Moretti, R. Santos and K. Yagyu, Light Charged Higgs bosons at the LHC in 2HDMs, Phys. Rev. D 84 (2011) 055028 [arXiv:1104.3178] [INSPIRE].

[10] LEP, DELPHI, OPAL, ALEPH and L3 collaborations, G. Abbiendi et al., Search for Charged Higgs bosons: Combined Results Using LEP Data, Eur. Phys. J. C 73 (2013) 2463 [arXiv: 1301.6065] [INSPIRE].

[11] CMS collaboration, Search for a light charged Higgs boson in top quark decays in pp collisions at $\sqrt{s}=7 \mathrm{TeV}$, JHEP 07 (2012) 143 [arXiv:1205.5736] [INSPIRE]. 
[12] ATLAS collaboration, Search for charged Higgs bosons decaying via $H^{+} \rightarrow \tau \nu$ in top quark pair events using pp collision data at $\sqrt{s}=7 \mathrm{TeV}$ with the ATLAS detector, JHEP 06 (2012) 039 [arXiv: 1204.2760] [INSPIRE].

[13] ATLAS collaboration, Search for charged Higgs bosons through the violation of lepton universality in $t \bar{t}$ events using pp collision data at $\sqrt{s}=7, T e V$ with the ATLAS experiment, JHEP 03 (2013) 076 [arXiv:1212.3572] [INSPIRE].

[14] CMS collaboration, Search for charged Higgs bosons with the $H^{+} \rightarrow \tau^{+} \nu_{\tau}$ decay channel in the fully hadronic final state at $\sqrt{s}=8 \mathrm{TeV}$, CMS-PAS-HIG-14-020 (2014) [INSPIRE].

[15] CMS collaboration, Search for a charged Higgs boson in pp collisions at $\sqrt{s}=8 \mathrm{TeV}$, JHEP 11 (2015) 018 [arXiv: 1508.07774] [INSPIRE].

[16] ATLAS collaboration, Search for charged Higgs bosons in the $\tau+$ jets final state with $p p$ collision data recorded at $\sqrt{s}=8 \mathrm{TeV}$ with the ATLAS experiment, ATLAS-CONF-2013-090 (2013) [INSPIRE].

[17] ATLAS collaboration, Search for charged Higgs bosons decaying via $H^{ \pm} \rightarrow \tau^{ \pm} \nu$ in fully hadronic final states using pp collision data at $\sqrt{s}=8 \mathrm{TeV}$ with the ATLAS detector, JHEP 03 (2015) 088 [arXiv: 1412.6663] [INSPIRE].

[18] J.G. Körner and M.C. Mauser, $O\left(\alpha_{s}\right)$ radiative corrections to polarized top decay into a charged Higgs $t(\uparrow) \rightarrow H^{+}+b$, Eur. Phys. J. C 54 (2008) 175 [hep-ph/0211098] [INSPIRE].

[19] A. Ali, E.A. Kuraev and Y.M. Bystritskiy, Radiatively corrected lepton energy distributions in top quark decays $t \rightarrow b W^{+} \rightarrow b\left(\ell^{+} \nu_{\ell}\right)$ and $t \rightarrow b H^{+} \rightarrow b\left(\tau^{+} \nu_{\tau}\right)$ and single charged prong energy distributions from subsequent $\tau^{+}$decays, Eur. Phys. J. C 67 (2010) 377 [arXiv:0911.3027] [INSPIRE].

[20] A. Czarnecki and S. Davidson, On the QCD corrections to the charged Higgs decay of a heavy quark, Phys. Rev. D 47 (1993) 3063 [hep-ph/9208240] [INSPIRE].

[21] J. Liu and Y.-P. Yao, QCD corrections to the charged Higgs boson decay of a heavy top quark, Phys. Rev. D 46 (1992) 5196 [hep-ph/9205245] [INSPIRE].

[22] C.S. Li and T.C. Yuan, QCD Correction to Charged Higgs Decay of the Top Quark, Phys. Rev. D 42 (1990) 3088 [Erratum ibid. D 47 (1993) 2156] [INSPIRE].

[23] S.M. Moosavi Nejad, B-mesons from top-quark decay in presence of the charged-Higgs boson in the Zero-Mass Variable-Flavor-Number Scheme, Phys. Rev. D 85 (2012) 054010 [arXiv:1110.1601] [INSPIRE].

[24] S.M. Moosavi Nejad, $\mathcal{O}\left(\alpha_{s}\right)$ corrections to the B-hadron energy distribution of the top decay in the Minimal Supersymmetric Standard Model considering GM-VFN scheme, Eur. Phys. J. C 72 (2012) 2224 [arXiv:1205.6139] [INSPIRE].

[25] S.M. Moosavi Nejad and S. Abbaspour, Next-to-leading order corrections to the spin-dependent energy spectrum of hadrons from polarized top quark decay in the general two Higgs doublet model, arXiv:1610.03811 [INSPIRE].

[26] N. Cabibbo, Unitary Symmetry and Leptonic Decays, Phys. Rev. Lett. 10 (1963) 531 [INSPIRE].

[27] M. Kobayashi and T. Maskawa, CP Violation in the Renormalizable Theory of Weak Interaction, Prog. Theor. Phys. 49 (1973) 652 [INSPIRE]. 
[28] B.A. Kniehl, G. Kramer and S.M. Moosavi Nejad, Bottom-Flavored Hadrons from Top-Quark Decay at Next-to-Leading order in the General-Mass Variable-Flavor-Number Scheme, Nucl. Phys. B 862 (2012) 720 [arXiv:1205.2528] [INSPIRE].

[29] S.M. Moosavi Nejad, Energy spectrum of bottom- and charmed-flavored mesons from polarized top quark decay $t(\uparrow) \rightarrow W^{+}+B / D+X$ at $O\left(\alpha_{s}\right)$, Phys. Rev. D 88 (2013) 094011 [arXiv:1310.5686] [INSPIRE].

[30] S.M. Moosavi Nejad and M. Balali, Angular analysis of polarized top quark decay into B-mesons in two different helicity systems, Phys. Rev. D 90 (2014) 114017 [Erratum ibid. D 93 (2016) 119904] [arXiv: 1409.1389] [INSPIRE].

[31] S.M. Moosavi Nejad, Spin-dependent energy distribution of B-hadrons from polarized top decays considering the azimuthal correlation rate, Nucl. Phys. B 905 (2016) 217 [arXiv: 1506.08871] [INSPIRE].

[32] S.M. Moosavi Nejad and M. Balali, Hadron energy spectrum in polarized top quark decays considering the effects of hadron and bottom quark masses, Eur. Phys. J. C 76 (2016) 173 [arXiv: 1602.05322] [INSPIRE].

[33] A. Ali, F. Barreiro and J. Llorente, Improved sensitivity to charged Higgs searches in Top quark decays $t \rightarrow b H^{+} \rightarrow b\left(\tau^{+} \nu_{\tau}\right)$ at the LHC using $\tau$ polarisation and multivariate tecnniques, Eur. Phys. J. C 71 (2011) 1737 [arXiv:1103.1827] [INSPIRE].

[34] J.C. Collins, Hard scattering factorization with heavy quarks: A General treatment, Phys. Rev. D 58 (1998) 094002 [hep-ph/9806259] [INSPIRE].

[35] V.D. Barger, J.L. Hewett and R.J.N. Phillips, New Constraints on the Charged Higgs Sector in Two Higgs Doublet Models, Phys. Rev. D 41 (1990) 3421 [InSPIRE].

[36] J. Binnewies, B.A. Kniehl and G. Kramer, Inclusive B meson production in $e^{+} e^{-}$and $p \bar{p}$ collisions, Phys. Rev. D 58 (1998) 034016 [hep-ph/9802231] [INSPIRE].

[37] V.N. Gribov and L.N. Lipatov, Deep inelastic ep scattering in perturbation theory, Sov. J. Nucl. Phys. 15 (1972) 438 [Yad. Fiz. 15 (1972) 781] [INSPIRE].

[38] G. Altarelli and G. Parisi, Asymptotic Freedom in Parton Language, Nucl. Phys. B 126 (1977) 298 [INSPIRE].

[39] Y.L. Dokshitzer, Calculation of the Structure Functions for Deep Inelastic Scattering and $e^{+} e^{-}$Annihilation by Perturbation Theory in Quantum Chromodynamics, Sov. Phys. JETP 46 (1977) 641 [Zh. Eksp. Teor. Fiz. 73 (1977) 1216] [inSPIRE].

[40] Particle Data Group collaboration, K. Nakamura et al., Review of particle physics, J. Phys. G 37 (2010) 075021 [inSPIRE].

[41] C. Degrande, R. Frederix, V. Hirschi, M. Ubiali, M. Wiesemann and M. Zaro, Accurate predictions for charged Higgs production: closing the $m_{H^{ \pm}} \sim m_{t}$ window, arXiv:1607.05291 [INSPIRE].

[42] B.A. Kniehl, G. Kramer, I. Schienbein and H. Spiesberger, Finite-mass effects on inclusive B meson hadroproduction, Phys. Rev. D 77 (2008) 014011 [arXiv: 0705.4392] [INSPIRE].

[43] OPAL collaboration, G. Abbiendi et al., Inclusive analysis of the $b$ quark fragmentation function in $Z$ decays at LEP, Eur. Phys. J. C 29 (2003) 463 [hep-ex/0210031] [INSPIRE].

[44] ALEPH collaboration, A. Heister et al., Study of the fragmentation of $b$ quarks into $B$ mesons at the Z peak, Phys. Lett. B 512 (2001) 30 [hep-ex/0106051] [INSPIRE]. 
[45] SLD collaboration, K. Abe et al., Precise measurement of the b-quark fragmentation function in $Z^{0}$ boson decays, Phys. Rev. Lett. 84 (2000) 4300 [hep-ex/9912058] [InSPIRE].

[46] SLD collaboration, K. Abe et al., Measurement of the b-quark fragmentation function in $Z^{0}$ decays, Phys. Rev. D 65 (2002) 092006 [Erratum ibid. D 66 (2002) 079905] [hep-ex/0202031] [INSPIRE].

[47] M. Soleymaninia, A.N. Khorramian, S.M. Moosavi Nejad and F. Arbabifar, Determination of pion and kaon fragmentation functions including spin asymmetries data in a global analysis, Phys. Rev. D 88 (2013) 054019 [Erratum ibid. D 89 (2014) 039901] [arXiv: 1306.1612] [INSPIRE].

[48] M. Soleymaninia, A.N. Khorramian and S.M. Moosavi Nejad, Pion fragmentation functions from $e^{+} e^{-}$production, J. Phys. Conf. Ser. 347 (2012) 012017 [INSPIRE].

[49] S.M. Moosavi Nejad, M. Soleymaninia and A.N. Khorramian, Global analysis of fragmentation functions and their application to polarized top quark decays considering new BABAR and Belle experimental data, Can. J. Phys. 95 (2017) 1 [arXiv:1402.7271] [INSPIRE].

[50] S.M. Moosavi Nejad, M. Soleymaninia and A. Maktoubian, Proton fragmentation functions considering finite-mass corrections, Eur. Phys. J. A 52 (2016) 316 [arXiv:1512.01855] [INSPIRE].

[51] J.C. Collins, Hard scattering factorization with heavy quarks: A General treatment, Phys. Rev. D 58 (1998) 094002 [hep-ph/9806259] [INSPIRE].

[52] G. Mahlon and S.J. Parke, Improved spin basis for angular correlation studies in single top quark production at the Tevatron, Phys. Rev. D 55 (1997) 7249 [hep-ph/9611367] [INSPIRE].

[53] J.H. Kühn, How to Measure the Polarization of Top Quarks, Nucl. Phys. B 237 (1984) 77 [INSPIRE].

[54] J.H. Kühn, A. Reiter and P.M. Zerwas, Z Decays to Top Quarks, Nucl. Phys. B 272 (1986) 560 [INSPIRE].

[55] S. Groote and J.G. Körner, Transverse polarization of top quarks produced in $e^{+} e^{-}$-annihilation at $O\left(\alpha_{s}\right)$, Z. Phys. C 72 (1996) 255 [Erratum ibid. C 70 (2010) 531] [hep-ph/9508399] [INSPIRE]. 